British Journal of Nutrition (2022), 127, 1018-1025

doi:10.1017/S000711452100180X

(C) The Author(s), 2021. Published by Cambridge University Press on behalf of The Nutrition Society. This is an Open Access article, distributed under the terms of the Creative Commons Attribution licence (http://creativecommons.org/licenses/by/4.0/), which permits unrestricted re-use, distribution, and reproduction in any medium, provided the original work is properly cited.

\title{
Iron-biofortified pearl millet consumption increases physical activity in Indian adolescent schoolchildren after a 6-month randomised feeding trial
}

\author{
Laura M. Pompano ${ }^{1,3}$, Sarah V. Luna ${ }^{1} \dagger$, Shobha A. Udipi ${ }^{2}$, Padmini S. Ghugre ${ }^{2}$, Eric M. Przybyszewski ${ }^{1}$ and \\ Jere Haas $^{1 *}$ \\ ${ }^{1}$ Cornell University, Savage Hall, Ithaca, NY 14853, USA \\ ${ }^{2}$ S.N.D.T. Women's University, 1, Nathibai Thackersey Road, Mumbai 400020, India \\ ${ }^{3}$ HarvestPlus, International Food Policy Research Institute, 1201 I St NW, Washington, DC 20005 USA
}

(Submitted 26 September 2020 - Final revision received 12 May 2021 - Accepted 21 May 2021 - First published online 3 June 2021)

\section{Abstract}

Fe deficiency has negative effects on voluntary physical activity (PA); however, the impact of consuming Fe-biofortified staple foods on voluntary PA remains unclear. This study compared the effects of consuming Fe-biofortified pearl millet or a conventional pearl millet on measures of voluntary PA in Indian schoolchildren (ages 12-16 years) during a 6-month randomised controlled feeding trial. PA data were collected from 130 children using Actigraph GT3X accelerometers for $6 \mathrm{~d}$ at baseline and endline. Minutes spent in light and in moderate-to-vigorous PA were calculated from accelerometer counts using Crouter's refined two-regression model for children. Mixed regression models adjusting for covariates were used to assess relationships between intervention treatment or change in Fe status and PA. Children who consumed Fe-biofortified pearl millet performed 22.3 (95\% CI 1.8, 42.8, P=0.034) more minutes of light PA each day compared with conventional pearl millet. There was no effect of treatment on moderate-to-vigorous PA. The amount of Fe consumed from pearl millet was related to minutes spent in light PA (estimate $3.4 \mathrm{~min} / \mathrm{mg} \mathrm{Fe}(95 \% \mathrm{CI} 0 \cdot 3,6 \cdot 5, P=0.031)$ ) and inversely related to daily sedentary minutes (estimate $-5.4 \mathrm{~min} / \mathrm{mg} \mathrm{Fe}(95 \% \mathrm{CI}-$ 9.9, $-0 \cdot 9, P=0 \cdot 020)$ ). Consuming Fe-biofortified pearl millet increased light PA and decreased sedentary time in Indian schoolchildren in a dose-dependent manner.

Key words: Adolescent health: Iron deficiency: Physical activity: Accelerometry: Iron biofortification: Pearl millet: Sedentary behaviour

Fe deficiency is the most common micronutrient deficiency in both developed and developing countries ${ }^{(1,2)}$. Previous literature has shown that improving the Fe status of Fe-deficient adults can increase their physical activity (PA) levels, with both factory workers and tea pickers showing improvements in their daily PA after receiving $\mathrm{Fe}$ supplements ${ }^{(3,4)}$. Additionally, Crouter et al. reported that women with healthy Fe levels performed 52.1 more $\mathrm{min} / \mathrm{d}$ of light PA (LPA) and spent 68.4 fewer $\mathrm{min} / \mathrm{d}$ in sedentary behaviours compared with women with poor Fe status ${ }^{(5)}$. These studies collectively suggest that resolving Fe deficiency in adults increases LPA - which has been shown to be beneficially associated with mortality, lipid and glucose metabolism, and obesity ${ }^{(6)}$ - and may also reduce sedentary behaviour, potentially as a result of Fe-deficiency-related fatigue $^{(7)}$

While interventions such as food fortification, dietary Fe supplementation or increasing dietary diversity are all effective strategies for addressing Fe deficiencies in many populations, these methods are often not feasible or accessible for the rural poor and other high-risk populations ${ }^{(8)}$. One method developed to reach these groups is biofortification, or the process of increasing the concentration and bioavailability of essential micronutrients in staple crops using conventional plant breeding and agronomic practices. The technique has the potential to become a sustainable, inexpensive and effective solution to Fe deficiency at the population level ${ }^{(9)}$. Pearl millet (Pennisetum glaucum) is a staple food in India, particularly in the states of Rajasthan, Gujarat and Maharashtra ${ }^{(10,11)}$, where this study took place. Previous work by our research group has demonstrated that the consumption of Fe-biofortified pearl millet was able to improve the $\mathrm{Fe}$ status and cognitive function of Indian children ${ }^{(12,13)}$. However, the effects of consuming an Fe-biofortified staple food on voluntary PA, which is known to be related to Fe deficiency ${ }^{(14-16)}$, remain unclear.

Abbreviations: LPA, light physical activity; PA, physical activity; Hb, hemoglobin; sFer, serum ferritin.

* Corresponding author: Jere D. Haas, email jdh12@cornell.edu

$\dagger$ Deceased 20 May 2019. 
While previous studies have focused on Fe-deficient adults, it is logical that a similar relationship could be observed in children; however, to date, the relationship between Fe status and voluntary $\mathrm{PA}$ in children remains poorly understood ${ }^{(12)}$. Therefore, the objective of this study was to examine the effect of consuming Fe-biofortified pearl millet during a 6-month randomised controlled feeding trial on objective measures of voluntary PA (time spent in light, moderate or vigorous PA as well as sedentary behaviours) in Indian schoolchildren aged 12-16 years. We hypothesised that participants who consumed Fe-biofortified pearl millet would increase their time spent performing non-sedentary PA compared with those who consumed standard variety pearl millet.

\section{Methods}

\section{Study participants}

This study was conducted from September 2011 to March 2012 among schoolchildren (12-16 years) in the Ahmednagar district, Maharashtra, India. Children were not eligible to participate if they: had severe anaemia (Hemoglobin, $\mathrm{Hb}<8.5 \mathrm{~g} / \mathrm{dl}$ ), were taking Fe supplements or any medication that could interfere with Fe absorption, had a chronic illness, if they or their parents or guardians were not willing to participate in the study or if they did not reside full time at the boarding school. All children were given anthelmintic treatments 4 weeks prior to the baseline blood collection and again at the study midpoint. Sample selection for the larger parent feeding trial was described in greater detail previously, but is summarised here ${ }^{(12)}$. Two hundred and eighty-eight male and female students were screened for inclusion in the parent feeding study. A total of 246 children were included in the parent analysis comparing the effect of consuming Fe-biofortified pearl millet (hereafter called 'Fe-PM') with consuming a popular commercial variety of pearl millet (hereafter called 'Control-PM') on changes in Fe status. Because it was not logistically possible to monitor PA for the entire sample of 246 subjects, a subsample of students with worst Fe status was selected to participate in the PA assessment by selecting the 130 participants with the lowest screening serum ferritin (sFer) values in order to understand the relationship between Fe status and PA among those with the greatest potential to benefit from additional dietary Fe. The flow of participants in the present analyses is shown in Fig. 1.

The study participants came from low-income households, lived at one of three hostels on the school campus and took all meals in the communal dining area of each hostel. Participants were selected because they represented an age and socio-economic segment of the Indian population at high risk for Fe deficiency ${ }^{(17)}$ and because their staple cereal was pearl millet. They regularly consumed large quantities of pearl millet (about $150-350 \mathrm{~g} / \mathrm{d}$, dried) in the form of bhakri, a type of round flatbread, during both lunch and dinner. Daily bhakri consumption was monitored and recorded for each subject by trained

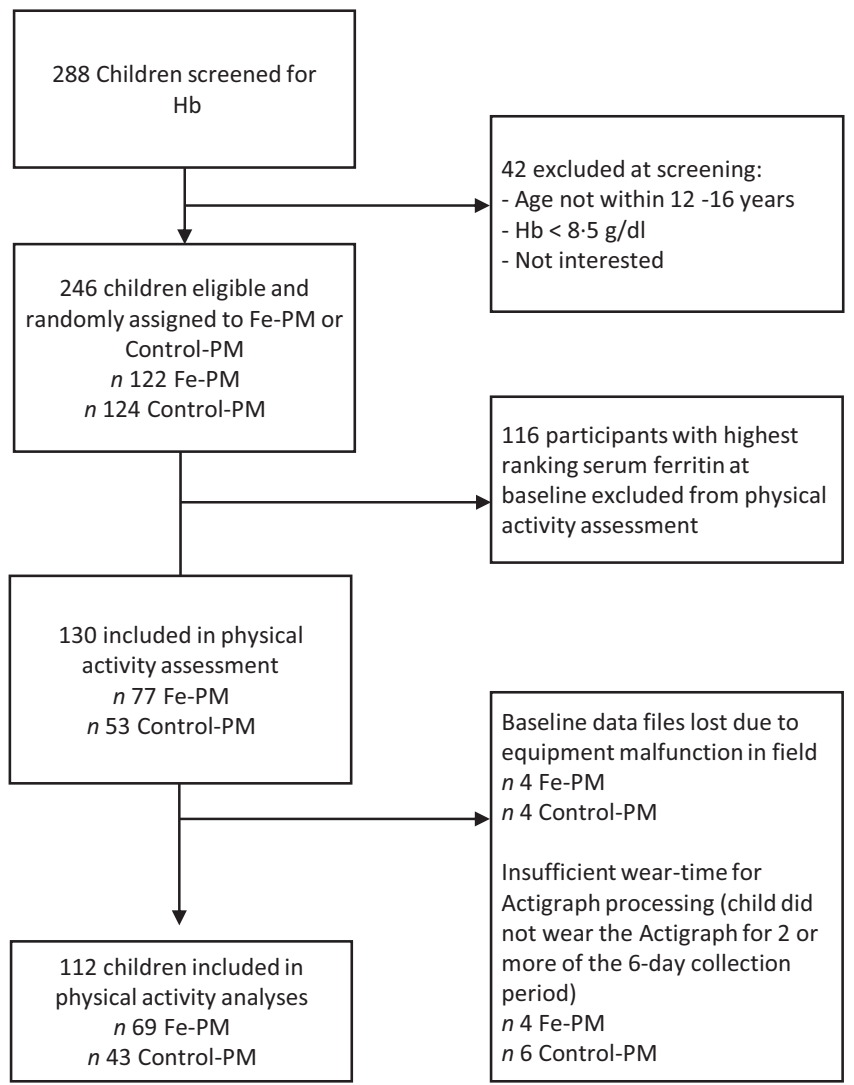

Fig. 1. CONSORT diagram of study participant selection. Fe-PM, treatment arm receiving iron-biofortified pearl millet; Control-PM, treatment arm receiving standard variety pearl millet; PA, physical activity. 
research assistants. All meals at each hostel were prepared in common kitchens. Dietary intake was assessed at baseline and endline using a 24-h recall administered by a trained research assistant. A food composition database was developed specifically for the diet of the children and was analysed using CS Dietary System software (CS Dietary System, version 1.1). The Fe content of bhakri was determined using ICP analyses of random bhakri samples collected from the study site every 2 weeks. Total Fe consumption was determined by multiplying the Fe per bhakri by the number of bhakri consumed. Details of the dietary intake protocol and analysis have been reported previously ${ }^{(12)}$.

\section{Study design}

The two treatments of pearl millet were randomly assigned at the individual level. The pearl millet flour was incorporated into the bhakri following a standardised local recipe. The bhakri made from Control-PM was identical in colour, taste and phytochemical composition and content as the bhakri from Fe-PM. Children were organised into feeding groups at mealtime (lunch and dinner) based on the type of bhakri they were to receive. There were two coded groups: one for Fe-PM and one for ControlPM. The distribution of bhakri was monitored by assistants who did not know which bhakri was assigned to each coded group. Each child's bhakri consumption (by quarters of a whole bhakri) was recorded by the assistants. Details of the parent study can be found in Finkelstein et al. ${ }^{(12)}$ The amount of Fe consumed from pearl millet increased for both the Fe-PM and Control-PM groups during the final 2 months (months 4-6) of the trial due to (1) a change in the variety of pearl millet fed to the Control-PM group and (2) the introduction of a pearl millet-based shev snack to all children. The Control-PM contained 21.8 parts per million $\mathrm{Fe}$, or $21.8 \mathrm{mg} \mathrm{Fe} / \mathrm{kg}$ pearl millet, from months 1 to 4 (variety DG9444), then increased to 52.1 parts per million in months 4-6 (variety JKBH778). The Fe-PM contained 86.3 parts per million Fe for the full 6-month intervention (variety ICTP8203). Children were given about 200-300 g of dry pearl millet per day through the bhakri, which was provided ad libitum.

\section{Physical activity protocol}

The present study's primary outcome was PA, which was assessed by accelerometers at baseline and after 6 months. PA data were collected in three sets of 40-45 subjects over 3 weeks at baseline and endline. The data were recorded in 10-s epochs using triaxial Actigraph GT3X accelerometers for six consecutive days from Tuesday through Sunday. No data were collected on Mondays, which were reserved for collecting the accelerometers, downloading data and recharging the batteries. Mondays were selected as the 'off day' of the week because they were similar to the other 4 school days in the week, when activities were likely to be similar. Movement was recorded in the transverse, frontal and sagittal planes. Trained research technicians placed the accelerometer affixed to an elastic belt worn over the child's left hip. Each child was instructed to wear the accelerometer at all times, except when sleeping or bathing. The raw count data were assessed for wear-time, with non-wear-time being determined using the Choi algorithm ${ }^{(18)}$. Insufficient wear-time was defined as the child not wearing the device for 2 or more days out of the 6-d collection period, with a valid day being defined as having a minimum of $10 \mathrm{~h}$ of wear-time. Metabolic equivalents were calculated from the accelerometer counts using Crouter's refined two-regression model for children with the R statistical package obtained from the Crouter research laboratory ${ }^{(19,20)}$. PA outcome variables included steps taken and number of minutes spent in sedentary behaviours like sitting in class (metabolic equivalent $=1$ ), light activity like walking, and performing chores or games (LPA, metabolic equivalent 1-3) and moderateand-vigorous activity like running or playing sports (metabolic equivalent $>3$ ).

\section{Laboratory analyses}

Blood draws were performed at baseline, after 4 months and at 6 months (endline) and assessed for the following blood biomarkers: Hb, sFer and soluble transferrin receptor as well as calculated total body Fe. Fe deficiency was defined as sFer $<15 \mu \mathrm{g} / \mathrm{l}$. Anaemia was defined as $\mathrm{Hb}<12.0 \mathrm{~g} / \mathrm{dl}$. Total body $\mathrm{Fe}$ was calculated as a ratio of soluble transferrin receptor:sFer using the formula reported by $\mathrm{Cook}^{(21)}$. All Fe biomarkers, $\alpha$-1-acid glycoprotein and C-reactive protein were analysed by Metropolis HealthCare Laboratory in Mumbai. Laboratory methods for blood analysis of Fe and inflammation biomarkers were described in detail previously ${ }^{(12)}$.

sFer was adjusted for inflammation using the regression correction approach described by the Biomarkers Reflecting Inflammation and Nutritional Determinants of Anemia project $^{(22)}$. For comparison, all analyses were also run without the Biomarkers Reflecting Inflammation and Nutritional Determinants of Anemia correction, both including and excluding participants with C-reactive protein $>3 \mathrm{mg} / \mathrm{l}$ and $\alpha$-1-acid glycoprotein $>1 \mathrm{~g} / \mathrm{l}$. No differences were observed in significance levels or the direction or magnitude of treatment effects between the corrected and uncorrected data. All ferritin values presented in the results and tables reflect the Biomarkers Reflecting Inflammation and Nutritional Determinants of Anemia-corrected values and do not exclude individuals with inflammation.

Anthropometry was performed at baseline and 6 months by trained research assistants as previously described ${ }^{(23)}$.

\section{Statistical analyses}

A sample size calculation, based on a previous study examining PA in Fe-deficient and Fe-replete populations ${ }^{(5)}$, showed that a sample size of 53/group would be sufficient to detect a 50.7 $\mathrm{min} / \mathrm{d}$ difference in LPA between groups assuming an $\alpha$ of 0.05 and $90 \%$ power. Descriptive statistics are expressed as means with $95 \%$ CI. One-way ANOVA was used to compare demographic and background characteristics between treatment groups. Tukey adjustments for multiple comparisons were used in ANOVA models with more than two groups. Linear regression models were used to analyse the treatment effects on endline $\mathrm{Hb}$, ferritin, soluble transferrin receptor, body Fe and PA variables. All models included baseline value and sex as covariates. Actigraph wear-time was included as a covariate in all analytical models examining PA outcomes, as recommended by Crouter et $a l^{(19,20)}$. Due to unforeseen problems in the study 
implementation, there were some limitations that may have reduced our ability to observe effects in the intention-to-treat analyses since most participants were consuming sufficient $\mathrm{Fe}$ by month 6 . Therefore, several secondary analyses were conducted to explore the biological plausibility of the results observed for treatment group differences. Secondary analyses included testing for changes in Fe status as a predictor of changes in PA using linear regression models, adjusting for the same covariates included in the analyses of intervention effects. An adjusted $P$-value of $<0.05$ was considered statistically significant for main effects, and a $P$-value of $<0 \cdot 10$ was considered statistically significant for interactions. No biomarker-by-sex interactions were observed in any secondary analysis model. All results of secondary analyses are presented with sexes combined, with sex included as a covariate. All analyses were done in SAS 9.4 software (SAS Institute Inc., Cary, NC, USA).

\section{Ethics}

This study was conducted according to the guidelines laid down in the Declaration of Helsinki, and all procedures involving human subjects/patients were approved by the Intersystem Biomedica Ethics Committee in Mumbai, India as well as the Institutional Review Boards of Cornell University, The University of Oklahoma and The Pennsylvania State University. Informed written consent was obtained from each participant as well as their guardians and the management and heads of the schools. This trial was registered at ClinicalTrials.gov as NCT02152150.

\section{Results}

Demographic information is shown in Table 1 . There was no difference in any baseline measure between the Fe-PM and Control-PM groups. The distribution of males and females in each group was not statistically different $\left(\chi^{2} P\right.$-value $\left.=0.49\right)$.

Table 1. Participant characteristics at baseline (Mean values and standard deviations)

\begin{tabular}{|c|c|c|c|c|}
\hline & \multicolumn{2}{|c|}{ Control-PM } & \multicolumn{2}{|c|}{ Fe-PM } \\
\hline & Mean & SD & Mean & SD \\
\hline$n$ & 43 & & 69 & \\
\hline \multicolumn{5}{|l|}{ Sex (\% male) } \\
\hline$\%$ & $55 \cdot 8$ & & $62 \cdot 3$ & \\
\hline Age (years) & $13 \cdot 8$ & 1.4 & $13 \cdot 6$ & 1.4 \\
\hline $\mathrm{Hb}(\mathrm{g} / \mathrm{dl})$ & $12 \cdot 4$ & $1 \cdot 0$ & $12 \cdot 5$ & 0.9 \\
\hline Ferritin $(\mu \mathrm{g} / \mathrm{l})$ & $15 \cdot 1$ & $8 \cdot 7$ & $18 \cdot 0$ & $10 \cdot 8$ \\
\hline $\mathrm{sTfR}(\mathrm{mg} / \mathrm{l})$ & $6 \cdot 6$ & $2 \cdot 3$ & $6 \cdot 8$ & $2 \cdot 4$ \\
\hline Body $\mathrm{Fe}(\mathrm{mg} / \mathrm{kg})$ & 1.3 & $2 \cdot 6$ & 1.9 & $2 \cdot 8$ \\
\hline AGP (mg/ml) & 0.6 & 0.2 & 0.6 & 0.2 \\
\hline $\mathrm{CRP}(\mathrm{mg} / \mathrm{l})$ & 0.4 & 0.7 & 1.0 & $3 \cdot 4$ \\
\hline LPA (min at $1-3 \mathrm{MET}$ ) & 251 & 56 & 237 & 52 \\
\hline MVPA (min at > 3 MET) & 95 & 47 & 96 & 48 \\
\hline Sedentary time (min) & 480 & 95 & 494 & 98 \\
\hline
\end{tabular}

Fe-PM, Fe-biofortified pearl millet; Control-PM, control pearl millet; sTfR, soluble transferrin receptor; AGP, $\alpha$-1-acid glycoprotein; CRP, C-reactive protein; LPA, light physical activity, defined as minutes spent performing activities in the 1-3 metabolic equivalents range; MVPA, moderate-to-vigorous physical activity, defined as minutes spend performing activities above 3 metabolic equivalents.
At baseline, $53.6 \%$ ( $n$ 60) of the PA sample participants were Fe-deficient (sFer $<15 \mu \mathrm{g} / \mathrm{l})$ and $34.5 \%$ were anaemic $(n$ 41, $\mathrm{Hb}<12.0 \mathrm{~g} / \mathrm{dl}$ ), of whom $58.5 \%$ ( $n$ 24) were Fe-deficient anaemic. Fourteen participants (11.8\%) had soluble transferrin receptor concentrations above $8.3 \mathrm{mg} / \mathrm{l}$, while twenty-nine children (24.4\%) had negative body Fe values. Additionally, 5.4\% ( $n$ 6) and $4.5 \%(n 5)$ of participants had inflammation based on $\alpha$-1-acid glycoprotein ( $>1 \mathrm{~g} / \mathrm{l})$ or C-reactive protein $(>3.0$ $\mathrm{mg} / \mathrm{l})$, respectively. There were no differences in the prevalence of inflammation by treatment group or sex at baseline.

The Control-PM group consumed 5.9 (SD 1.1) $\mathrm{mg} \mathrm{Fe} / \mathrm{d}$ and 9.7 (sD 1.1) $\mathrm{mg} \mathrm{Fe} / \mathrm{d}$ from the pearl millet during months 1-4 and 5-6, respectively. The Fe-PM group consumed 10.7 (sD 2.3) $\mathrm{mg} \mathrm{Fe} / \mathrm{d}$ and $16.5 \mathrm{mg} \mathrm{Fe} / \mathrm{d}$ in months $1-4$ and $5-6$, respectively. The Fe-PM group consumed $4.5 \mathrm{mg} / \mathrm{d}$ more Fe than the Control-PM group from months 1 to 4 (one-way ANOVA $P$ value $<0.001)$ and $5.6 \mathrm{mg} / \mathrm{d}$ more $\mathrm{Fe}$ than the Control-PM group from months 5 to 6 (one-way ANOVA $P$ value $<0.001)$. The number of bhakri consumed each day did not differ between the treatment groups, with the ControlPM group consuming 1.1 (SD 0.3) bhakri/d and the Fe-PM group consuming $1 \cdot 2(\mathrm{SD} 0 \cdot 2)$ bhakri per day (one-way ANOVA $P$ value $=0 \cdot 40)$

\section{Effect of treatment on iron status}

No effects of treatment on Fe status were observed at 6 months in the present study ( $n$ 112), but the Fe-PM group had significantly higher body Fe than the Control-PM group at 4 months, on the basis of linear mixed models, controlling for baseline Fe status and sex (Table 2). There were no significant changes in any Fe status measure between 4 and 6 months for either treatment group.

\section{Effect of treatment on physical activity}

Table 3 shows the results of the analyses of the two dietary treatments on PA outcomes at 6 months. No treatment-by-sex interactions were observed for any intention-to-treat model; therefore, results are presented with both sexes combined; however, sex was retained as a covariate in all models. There were no differences in wear-time between treatment groups at baseline (Fe-PM: 827 (sD 79) min, Control-PM: 825 (sD 108) min, oneway ANOVA $P=0.91$ ) or at month 6 (Fe-PM: 823 (sD 104) min, Control-PM: 811 (sD 125) min, one-way ANOVA $P=0.59$ ).

There was a significant effect of treatment on minutes spent in LPA, with the Fe-PM group performing $22.3 \mathrm{~min} / \mathrm{d}$ (95\% CI 1.8, $42.8 \mathrm{~min} / \mathrm{d}$ ) more LPA than the Control-PM group (linear mixed model $P=0.034$ ). No treatment effects were observed for minutes per day in sedentary behaviour, moderate-and-vigorous activity or steps.

\section{Secondary analyses}

First, we examined whether the daily average amount of Fe consumed from pearl millet during the 6-month study differed by treatment group. The treatment group significantly predicted the amount of $\mathrm{Fe}$ consumed per day. Participants in the Control-PM group consumed an average $7.3 \mathrm{mg} / \mathrm{d}$ (95\% CI 
Table 2. Iron status at months 4 and 6 , adjusting for baseline status and sex (Mean values and $95 \%$ confidence intervals)

\begin{tabular}{|c|c|c|c|c|c|c|c|c|c|}
\hline \multirow[b]{2}{*}{ Time point } & \multirow[b]{2}{*}{ Outcome } & \multirow[b]{2}{*}{$n$} & \multicolumn{2}{|c|}{ Control-PM } & \multicolumn{2}{|c|}{ Fe-PM } & \multicolumn{2}{|c|}{ Diff (Control-Fe) } & \multirow[b]{2}{*}{ Treatment $P$ value } \\
\hline & & & Mean & $95 \% \mathrm{Cl}$ & Mean & $95 \% \mathrm{Cl}$ & Mean & $95 \% \mathrm{Cl}$ & \\
\hline \multirow[t]{5}{*}{ Month 4} & $\mathrm{Hb}(\mathrm{g} / \mathrm{dl})$ & 102 & $13 \cdot 8$ & $12 \cdot 2,15 \cdot 4$ & $13 \cdot 8$ & $12 \cdot 2,15 \cdot 3$ & $-0 \cdot 1$ & $-0.4,0.2$ & 0.96 \\
\hline & sFer $(\mu \mathrm{g} / \mathrm{l})$ & 91 & $12 \cdot 4$ & $5 \cdot 3,19 \cdot 6$ & $16 \cdot 3$ & $11 \cdot 3,21 \cdot 3$ & -3.9 & $-12 \cdot 7,5 \cdot 0$ & 0.39 \\
\hline & Log sFer & 91 & $2 \cdot 3$ & $2 \cdot 0,2 \cdot 7$ & $2 \cdot 4$ & $2 \cdot 1,2 \cdot 7$ & -0.1 & $-0.4,0.2$ & 0.52 \\
\hline & $\mathrm{sTfR}(\mathrm{mg} / \mathrm{l})$ & 91 & $7 \cdot 7$ & $6 \cdot 6,8.8$ & $7 \cdot 2$ & $6 \cdot 3,8 \cdot 2$ & 0.5 & $-0.4,1.3$ & 0.31 \\
\hline & $\mathrm{BI}$ & 91 & $1 \cdot 3$ & $0.6,2.0$ & $2 \cdot 3$ & $1.7,2.9$ & -1.0 & $-1.9,-0.1$ & 0.036 \\
\hline \multirow[t]{5}{*}{ Month 6} & $\mathrm{Hb}(\mathrm{g} / \mathrm{dl})$ & 98 & $12 \cdot 4$ & $12 \cdot 1,12 \cdot 7$ & 12.5 & $12 \cdot 2,12 \cdot 7$ & $-0 \cdot 1$ & $-0.4,0.3$ & 0.75 \\
\hline & Fer $(\mu \mathrm{g} / \mathrm{l})$ & 99 & $19 \cdot 6$ & $14 \cdot 6,24 \cdot 5$ & $20 \cdot 1$ & $15 \cdot 7,24 \cdot 6$ & -0.5 & $-4 \cdot 6,3 \cdot 6$ & 0.79 \\
\hline & Log Fer & 99 & $2 \cdot 7$ & $2 \cdot 4,3 \cdot 1$ & $2 \cdot 8$ & $2 \cdot 5,3 \cdot 1$ & -0.1 & $-0.2,0.1$ & 0.60 \\
\hline & $\mathrm{sTfR}(\mathrm{mg} / \mathrm{l})$ & 99 & $7 \cdot 1$ & $6 \cdot 2,8 \cdot 1$ & $7 \cdot 1$ & $6 \cdot 3,8 \cdot 0$ & -0.0 & $-0.7,0.7$ & 0.93 \\
\hline & $\mathrm{BI}$ & 99 & $2 \cdot 2$ & $1 \cdot 2,3 \cdot 2$ & 2.5 & $1.6,3.4$ & -0.3 & $-1 \cdot 1,0.6$ & 0.51 \\
\hline
\end{tabular}

Control-PM, group receiving control pearl millet; Fe-PM, group receiving Fe-biofortified pearl millet; Diff, difference in LS means for Control-PM and Fe-PM groups; sFer, serum ferritin; sTfR, soluble transferrin receptor; Bl, body Fe.

${ }^{*}$ Results of linear mixed models adjusted for baseline value of outcome and sex with a random effect of hostel (location).

Table 3. Analysis of treatment group differences for physical activity at 6 months* (Mean values and $95 \%$ confidence intervals)

\begin{tabular}{|c|c|c|c|c|c|c|c|c|}
\hline \multirow[b]{2}{*}{ Daily physical activity } & \multirow[b]{2}{*}{$n$} & \multicolumn{2}{|c|}{ Control-PM } & \multicolumn{2}{|c|}{ Fe-PM } & \multicolumn{2}{|c|}{ Diff (Ctl - Fe) } & \multirow[b]{2}{*}{ TRT $P$ value } \\
\hline & & Mean & $95 \% \mathrm{Cl}$ & Mean & $95 \% \mathrm{Cl}$ & Mean & $95 \% \mathrm{Cl}$ & \\
\hline Sedentary time (min) & 112 & 499 & 475,523 & 475 & 455,494 & $24 \cdot 4$ & $-6 \cdot 6,55 \cdot 3$ & 0.12 \\
\hline LPA (min) & 112 & 238 & 212,263 & 260 & 237,283 & $-22 \cdot 3$ & $-42 \cdot 8,-1 \cdot 8$ & 0.034 \\
\hline MVPA (min) & 112 & 90 & 78,103 & 88 & 78,98 & 1.9 & $-14 \cdot 2,17 \cdot 9$ & 0.82 \\
\hline Steps & 112 & 9951 & 8956,10947 & 9909 & 9086,10731 & 42 & $-1110,1196$ & 0.94 \\
\hline
\end{tabular}

Control-PM, group receiving control pearl millet; Fe-PM, group receiving Fe-biofortified pearl millet; Diff (Ctl - Fe), difference in LS means between Control-PM and Fe-PM groups; TRT $P$ value, $P$ value for treatment group; LPA, light physical activity, defined as minutes spent performing activities in the 1-3 metabolic equivalents range (METS); MVPA, moderateto-vigorous physical activity, defined as minutes spend performing activities above 3 METS.

${ }^{*}$ Results of linear mixed models adjusted for baseline value of outcome, sex, as well as baseline and endline wear-time. All models include a random effect of hostel (location) and are adjusted for multiple comparisons using a Tukey adjustment.

$5 \cdot 8,8 \cdot 7 \mathrm{mg} / \mathrm{d}$ ) over the 6-month trial, while participants in the Fe-PM group consumed an average of $12 \cdot 8 \mathrm{mg} / \mathrm{d}(95 \% \mathrm{CI}$ $11 \cdot 4,14.2 \mathrm{mg} / \mathrm{d}), P<0 \cdot 001$.

Second, we examined whether the average amount of Fe consumed per day over the study duration was related to endline PA, adjusting for baseline, sex and wear-time. Participants who consumed more Fe during the study engaged in a greater number of minutes of voluntary LPA and had fewer sedentary minutes, regardless of treatment group (Fig. 2). We also examined whether baseline to endline changes in Fe biomarker concentrations were related to endline PA (adjusting for baseline performance). No significant relationships were observed between changes in any Fe biomarker and any PA outcome (data not shown).

In order to examine the internal consistency of the PA data, we also examined whether the change in LPA minutes was related to change in sedentary time. While treatment group did not impact this relationship, there was a strong inverse relationship between the change in minutes spent performing LPA and minutes spent in sedentary behaviours (effect estimate and $\left.95 \% \mathrm{CI}-1.3 \mathrm{~min}(-1.4,-1.1), P<0.001, R^{2}=0.85\right)$

Finally, we examined the effect of baseline Fe status (Fe-deficient non-anaemic, Fe-deficient anaemia, Fe replete non-anaemic, Fe replete anaemic) on all PA outcome variables. There were no significant differences in any outcome measure related to the Fe status groups at baseline on the basis of one-way ANOVA adjusted for pairwise comparisons using a Tukey correction (data not shown).

\section{Discussion}

The goal of the present study was to determine the effects of consuming Fe-biofortified pearl millet on voluntary PA. The study showed that children who consumed Fe-biofortified pearl millet performed 22.3 min more LPA per day than those who consumed control pearl millet. Specifically, this LPA appears to replace sedentary time, as evidenced by the roughly equivalent, though not statistically significant, decrease in sedentary time in the Fe-PM group. The effect of the intervention was further supported by the relationship between the amount of Fe consumed from pearl millet during the intervention and the changes in sedentary and LPA behaviours, with greater Fe intake being related to more minutes in LPA and fewer sedentary minutes

This is the first study to show that consumption of an Fe-biofortified staple food increases LPA compared with the consumption of a conventional variety of the staple. This is also the first study to examine PA outcomes in a biofortification intervention in children. The current findings are supported by those of previous research, which have shown that adults with poorer 


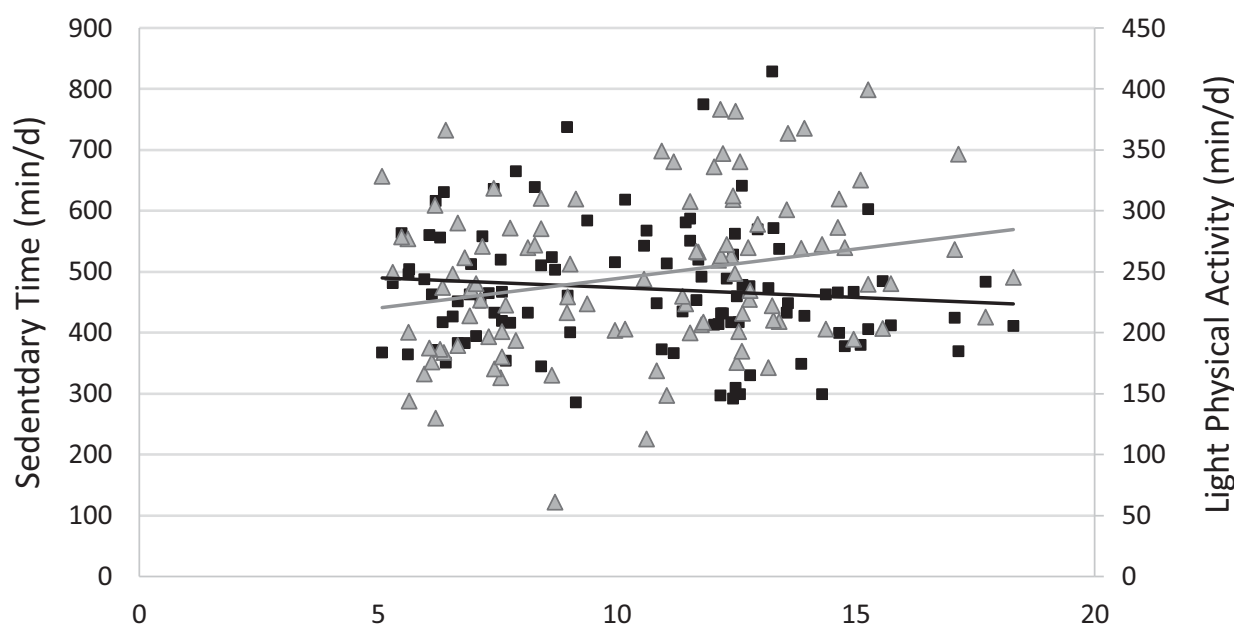

Average iron consumed per day from pearl millet ${ }^{2}(\mathrm{mg})$

\begin{tabular}{lll} 
Outcome & Effect estimate $(95 \% \mathrm{Cl})$ & $\boldsymbol{P}$ value \\
\hline Sedentary Time & $-5 \cdot 4(-9 \cdot 9,-0 \cdot 9)$ & 0.020 \\
Light Physical Activity & $3.4(0 \cdot 3,6 \cdot 5)$ & 0.031
\end{tabular}

Fig. 2. Minutes spent in light physical activity and sedentary behaviour predicted by average iron consumption ${ }^{1} .{ }^{1}$ Results of linear mixed models adjusted for baseline value of outcome, sex and (for physical activity variables) baseline and endline wear-time. All models include a random effect of hostel (location) and are adjusted for multiple comparisons using a Tukey adjustment. ${ }^{2}$ Refers to the amount of iron consumed from pearl millet over the 6-month study duration and not iron from other dietary sources. $\square$, Sedentary; $\triangle$, Light Physical Activity.

Fe status had increased sedentary time compared with those with normal Fe levels ${ }^{(5)}$. Another study found that adult women who consumed Fe supplements performed 30 min more daily non-sedentary PA than those supplemented with placebo $^{(4)}$, which is similar to the 22 min additional LPA observed in the present study.

Furthermore, the number of minutes children spent in sedentary behaviours was strongly and inversely related to the number of minutes they spent performing LPA, suggesting that consumption of Fe-biofortified pearl millet leads children to replace roughly 22 min of sedentary time with LPA per day. This finding is further supported by our secondary analyses, which showed that the amount of Fe consumed from pearl millet, regardless of treatment group, was directly related to LPA and inversely related to sedentary time in a dose-dependent manner. Specifically, for each additional $1 \mathrm{mg} / \mathrm{d}$ of Fe consumed from pearl millet, participants performed 5 fewer min of sedentary activity and 3 additional min of LPA each day.

The dose-dependent response observed in the present study further supports the use of biofortification as a tool to address Fe deficiency in areas with high prevalence of Fe deficiency that are not served by Fe interventions such as supplementation or fortification. While it would be ideal to provide the full recommended daily $\mathrm{Fe}$ requirement in an $\mathrm{Fe}$ intervention where possible, the results of the present study suggest that replacing conventional pearl millet varieties with Fe-biofortified varieties could impact children's PA behaviours even if they provide only a portion of the recommended daily intake.

While the physiological mechanism between the consumption of Fe-biofortified crops and PA behaviours remains somewhat unclear, previous research suggests that fatigue may be a contributing factor. A recent meta-analysis showed that Fe supplementation improved self-reported fatigue in women with $\mathrm{Fe}$ deficiency without anaemia ${ }^{(7)}$. Similarly, a review by McClung \& Murray-Kolb also cite fatigue as an explanation for the observed relationship between Fe status and PA observed in previous studies $^{(4,5,16)}$. Further research should directly examine the results of Fe biofortification interventions on measures of fatigue to explore the mediating role it may have on voluntary PA.

Increased PA (and reduced sedentary time) has been shown to contribute to improved cognitive performance ${ }^{(16,24)}$, executive function $^{(25)}$, academic achievement and motor skills in children ${ }^{(24,26)}$. Additionally, a recent systematic review found that while there is currently a lack of high-quality data specifically looking at the health effects of LPA, existing studies have shown that increased LPA was favourably associated with diastolic blood pressure, insulin resistance and HDL-cholesterol in children and adolescents ${ }^{(27)}$. Another review using isotemporal substitution models found that reallocation of even $30 \mathrm{~min}$ of daily sedentary behaviour to LPA or more intense PA was associated with reduced risk of mortality and other health benefits ${ }^{(28)}$. Therefore, increasing daily PA and reducing sedentary behaviours through the consumption of biofortified pearl millet could not only improve the overall activity level of this population but could also lead to healthier development in other key aspects of child growth. Indeed, cognitive function was also shown to improve with the consumption of Fe-biofortified pearl millet in this same study population ${ }^{(13)}$.

This study had several limitations. The first is that the intervention effects on Fe status occurred at 4 months, while PA was only assessed at 6 months for logistic reasons. At 4 months, the Control-PM group was switched to a pearl millet variety with 
a higher Fe content and both groups received a pearl milletbased snack. These changes resulted in increased dietary Fe intake leading to improvements in Fe status in the Control-PM group between months 4 and $6^{(12)}$. Without PA measures at 4 months, it was not possible to determine if there was a significant intervention effect on PA at month 4 . We considered the possibility that the PA response at 6 months lagged after adequate Fe status was achieved at 4 months. However, there were no significant relationships that supported an effect. In addition, we did not directly measure fractional absorption of Fe from pearl millet. Rather, we assumed a fractional absorption of $\mathrm{Fe}$ of $7.5 \%$ from pearl millet based on a previous study ${ }^{(29)}$. Given the ICP analyses and the total number of bhakri consumed, the absorbed $\mathrm{Fe}$ values at endline were estimated to be approximately $64 \%$ and $109 \%$ of the USA estimated average requirement for this age group and weight $(15 \cdot 1 \mathrm{mg} / \mathrm{d}$ for adolescents 12-19 years of age), in the Control-PM and Fe-PM groups, respectively ${ }^{(12,30)}$. We also cannot exclude the possibility that there were differences in other nutrients between the control and high-Fe pearl millet that may have contributed to the results. Despite these limitations, a significant treatment effect was still observed for LPA at 6 months.

Additionally, the sample size of the PA subgroup was smaller than intended (Fig. 1). This was a result of eight lost data files ( 4 Fe-PM, 4 Control-PM), during transfer of data from the field site. These data are considered to be 'missing completely at random' and are not expected to introduce bias into the analyses. Data from another ten subjects (4 Fe-PM, 6 Control-PM) were lost at the analysis phase because they did not wear the Actigraph for 2 or more days, with a valid 'day' being defined as having a minimum of $10 \mathrm{~h}$ of wear time. These ten children did not differ from the greater sample in age, Fe status measures, inflammation, amount of bhakri consumed or any other variable tested (on the basis of one-way ANOVA, data not shown). Because these unusable data were distributed fairly evenly between the treatment groups and constituted $<10 \%$ of the overall sample, they were not likely to introduce to bias in a particular direction.

Finally, because this analysis used the participants with the lowest Fe status at baseline, the results of this study ( $n$ 112) may be less generalisable than if we had been able to assess the full sample from the parent study ( $n$ 246). This analysis was conducted as part of an efficacy study to determine the potential for using Fe-biofortified pearl millet to improve Fe status, with PA as a secondary outcome, in Indian schoolchildren and the results should be interpreted accordingly. Future research should investigate whether consuming additional $\mathrm{Fe}$ through dietary change in real-world conditions, such as those in a large, population-wide effectiveness study, show similar results on LPA, sedentary behaviour and other aspects of life related to PA such as fitness, fatigue, cognition and general health.

\section{Conclusions}

Understanding whether and how micronutrient deficiencies affect children's PA patterns in relation to their health is a topic that needs further exploration. This study is one of the first to use objective measures of PA and micronutrient status in a randomised controlled trial and is the first to demonstrate the effects of consuming an Fe-biofortified staple food on PA in children. The results of this study show that consuming Fe-biofortified pearl millet compared with conventional pearl millet increased LPA by $22 \mathrm{~min} / \mathrm{d}$ Indian schoolchildren. In addition, this relationship appeared to be dose-dependent, with children showing increased LPA and reduced sedentary behaviours as they consumed greater amounts of Fe from pearl millet each day. These changes could subsequently contribute to improved health indicators, healthier motor and cognitive development, and reduced risk of mortality in this population.

\section{Acknowledgements}

The authors would like to thank Dr. Scott Crouter for providing the R statistical package used to analyse the Actigraph data and his assistance in using it. We would also like to thank Brandan Denvir for his assistance with the early stages of data processing and analysis.

This study was made possible with support from HarvestPlus. HarvestPlus' principal donors are the UK Government; the Bill \& Melinda Gates Foundation; the USA Government's Feed the Future initiative; the Canadian Government; the European Commission; and donors to the CGIAR Research Program on Agriculture for Nutrition and Health (A4NH). HarvestPlus is also supported by the Children's Investment Fund Foundation and the John D. and Catherine T. MacArthur Foundation. HarvestPlus/IFPRI did not contribute to the design, implementation, analyses or writing of this study.

J. D. H., S. A. U. and P. S. G. designed the research; E. M. P. and S. V. L. collected the data; S. V. L. and L. M. P. analysed data; and L. M. P., J. D. H. and S. V. L. wrote paper. J. D. H. had primary responsibility for the final content. All authors read and approved the final version. The family of S. V. L. have given their approval for her inclusion as an author on this manuscript.

S. V. L., S. A. U., P. S. G. and E. M. P. claim no conflict of interest. J. D. H. and L. M. P. served as a paid technical consultant for HarvestPlus/IFPRI. At the time that the study was conducted, L. M. P. had no affiliation with HarvestPlus/IFPRI; however, L. M. P. finalised the manuscript preparation after the untimely death of S. V. L. while serving as a technical consultant for HarvestPlus/IFPRI.

\section{References}

1. International Nutritional Anemia Consultative Group, World Health Organization \& United Nations Childrens Fund (1998) Guidelines for the use of iron supplements to prevent and treat iron deficiency anemia. World Health Organization. https:// www.who.int/nutrition/publications/micronutriets/guidelines_ for_Iron_supplementation.pdf (accessed March 2020).

2. World Health Organization (2006) Guidelines on food fortification with micronutrients. https://www.who.int/publications/i/ item/9241594012 (accessed March 2020).

3. Edgerton VR, Gardner GW, Ohira Y, et al (1979) Irondeficiency anaemia and its effect on worker productivity and activity patterns. Br Med J 2, 1546-1549 
4. Li R, Chen X, Yan H, et al (1994) Functional consequences of iron supplementation in iron-deficient female cotton mill workers in Beijing, China. Am J Clin Nutr 59, 908-913

5. Crouter SE, DellaValle DM \& Haas JD (2012) Relationship between physical activity, physical performance, and iron status in adult women. Appl Physiol Nutr Metab 37, 697-705.

6. Füzéki E, Engeroff T \& Banzer W (2017) Health benefits of lightintensity physical activity: a systematic review of accelerometer data of the National Health and Nutrition Examination Survey (NHANES). Sport Med 47, 1769-1793.

7. Houston BL, Hurrie D, Graham J, et al (2018) Efficacy of iron supplementation on fatigue and physical capacity in non-anaemic iron-deficient adults: a systematic review of randomised controlled trials. BMJ Open 8, e019240.

8. Bouis HE \& Saltzman A (2017) Improving nutrition through biofortification: a review of evidence from HarvestPlus, 2003 through 2016. Glob Food Sec 12, 49-58.

9. Bouis HE, Low J, Mcewan M, et al. (2014) Biofortification: evidence and lessons learned linking agriculture and nutrition. http://www.fao.org/3/as660e/as660e.pdf (accessed March 2020).

10. Velu G, Rai K \& Sahrawat K (2008) Variability for grain iron and zinc content in a diverse range of pearl millet populations. J Crop Improv 35, 186-191

11. Parthasarathy Rao P, Birthal P, Reddy BV, et al (2006) Diagnostics of sorghum and pearl millet grains-based nutrition in India. Int Sorghum Millets Newsl 47, 93-96

12. Finkelstein JL, Mehta S, Udipi SA, et al (2015) A randomized trial of iron-biofortified pearl millet in school children in India.J Nutr 145, 1576-1581.

13. Scott SP, Murray-Kolb LE, Wenger MJ, et al (2018) Cognitive performance in Indian school-going adolescents is positively affected by consumption of iron-biofortified pearl millet: a 6month randomized controlled efficacy trial. J Nutr 1, 1462-1471.

14. Haas JD \& Brownlie T (2001) Iron deficiency and reduced work capacity: a critical review of the research to determine a causal relationship. J Nutr 131, 676S-688S

15. Pasricha S-R, Mchugh K \& Drakesmith H (2016) Regulation of hepcidin by erythropoiesis: the story so far. Annu Rev Nutr $\mathbf{3 6}$, 417-434.

16. McClung JP \& Murray-Kolb LE (2013) Iron nutrition and premenopausal women: effects of poor iron status on physical and neuropsychological performance. Annu Rev Nutr 33, 271-288.

17. International Institute for Population Sciences (2013) Comprehensive Nutrition Survey in Maharashtra. https:// motherchildnutrition.org/india/pdf/IIPS-CNSM-Survey-Report. pdf (accessed April 2020).
18. Choi L, Liu Z, Matthews CE, et al. (2011) Validation of accelerometer wear and nonwear time classification algorithm. Med Sci Sports Exerc 43, 357-364.

19. Crouter SE, Kuffel E, Haas JD, et al (2010) Refined two-regression model for the ActiGraph accelerometer. Med Sci Sports Exerc 42, 1029-1037.

20. Crouter SE, Horton M \& Bassett DR (2012) Use of a two-regression model for estimating energy expenditure in children. Med Sci Sports Exerc 44, 1177-1185.

21. Cook JD, Flowers CH \& Skikne BS (2003) The quantitative assessment of body iron. Blood 101, 3359-3364.

22. Namaste SM, Rohner F, Huang J, et al (2017) Adjusting ferritin concentrations for inflammation: biomarkers Reflecting Inflammation and Nutritional Determinants of Anemia (BRINDA) project. Am J Clin Nutr 106, 359S-371S.

23. Savanur MS, Sathye A, Udawant A, et al (2017) Nutritional status and physical fitness of tribal adolescents in ahmednagar district of Maharashtra. Ecol Food Nutr 56, 552-566.

24. Donnelly JE, Hillman CH, Castelli D, et al (2016) Physical activity, fitness, cognitive function, and academic achievement in children: a systematic review. Med Sci Sports Exerc 48, $1197-1222$

25. Scott SP \& Murray-Kolb LE (2016) Iron status is associated with performance on executive functioning tasks in nonanemic young women. J Nutr 146, 30-37.

26. Zeng N, Ayyub M, Sun H, et al (2017) Effects of physical activity on motor skills and cognitive development in early childhood: A systematic review. Biomed Res Int 2017, 2760716.

27. Poitras VJ, Gray CE, Borghese MM, et al (2016) Systematic review of the relationships between objectively measured physical activity and health indicators in school-aged children and youth. Appl Physiol Nutr Metab 41, S197-S239.

28. Grgic J, Dumuid D, Bengoechea EG, et al (2018) Health outcomes associated with reallocations of time between sleep, sedentary behaviour, and physical activity: a systematic scoping review of isotemporal substitution studies. Int $J$ Behav Nutr Phys Act 15, 69.

29. Cercamondi CI, Egli IM, Mitchikpe E, et al (2013) Total iron absorption by young women from iron-biofortified pearl millet composite meals is double that from regular millet meals but less than that from post-harvest iron-fortified millet meals. J Nutr 143, 1376-1382.

30. U.S. Department of Health and Human Services \& U.S. Department of Agriculture (2015) Dietary Guidelines for Americans 2015-2020. Eighth Edition. https://health.gov/ sites/default/files/2019-09/2015-2020_Dietary_Guidelines.pdf (accessed March 2020). 